\title{
Retraction: erbB expression changes in ethanol and 7,12- dimethylbenz (a) anthracene-induced oral carcinogenesis. Med Oral Patol Oral Cir Bucal. 2013 Mar 1;18(2):e325-31
}

\author{
Alejandro García Carranca, Edgar Zenteno Galindo, Maria Dolores Jiménez Farfán, Juan Carlos Hernán- \\ dez Guerrero
}

Correspondence:

Facultad de Odontologia de la UNAM, Mexico

farfanmd@unam.mx

Received: $28 / 11 / 2013$

Accepted: 03/12/2013
Garcia Carrancá A, Zentero Galindo E, Jiménez Farfán M.-D, Hernandez Guerrero J.-C. Retraction: erbB expression changes in ethanol and 7,12- dimethylbenz (a)anthracene-induced oral carcinogenesis. Med Oral Patol Oral Cir Bucal. 2013 Mar 1;18(2):e325-31. Med Oral Patol Oral Cir Bucal. 2014 Jan 1;19(1):e98.

http://www.medicinaoral.com/medoralfree01/v19i1/medoralv19i1p98.pdf

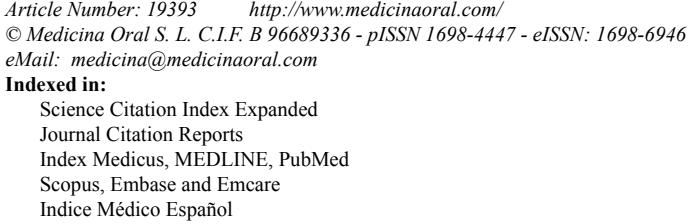

\begin{abstract}
The authors (Garcia Carrancá A, Zentero Galindo E, Jiménez Farfán MD and Hernandez Guerrero JC) express that one of the figures of the original article (Jacinto-Alemán LF, García-Carrancá A, LeybaHuerta ER, Zenteno-Galindo E, Jiménez-Farfán MD, Hernández-Guerrero JC. erbB expression changes in ethanol and 7,12- dimethylbenz (a)anthracene-induced oral carcinogenesis. Med Oral Patol Oral Cir Bucal. 2013 Mar 1;18(2):e325-31.) corresponding to Western blots have not been found and the voluntary alteration of this figure is evident.

The coauthors Alejandro García Carranca, Edgar Zenteno Galindo, Maria Dolores Jiménez Farfán and Juan Carlos Hernández Guerrero have made the decision to take back what has been published, as they have come to the conclusion, that at least this result is false.

The editor declare that the journal had the signed copyright by the authors when the article was initially published. This copyright document certifies that the undersigned authors warrants that the article is original; is not under consideration by another publication; and its tables or figures have not been previously published. The authors confirmed that the final article had been read and each author's contribution had been approved by the appropriate author. The editor has made the decision to retract the article due to the above comments of some authors against the rest. The editors apologize to the readers and reviewers of Med Oral Patol Oral Cir Bucal for the inconvenience caused by the authors of the article.
\end{abstract}

